\title{
IMPLICAÇÕES DA SÍNDROME DE BURNOUT EM PROFISSIONAIS DA SAÚDE DURANTE A PANDEMIA DE COVID-19: UMA REVISÃO INTEGRATIVE
}

\section{ARTIGO DE REVISÃO}

RIBEIRO, Yana Sarah Fernandes Souza ${ }^{1}$

LOPES, Rafael Nobre ${ }^{2}$

BRITO, Francisca Letícia Timbó ${ }^{3}$

SILVA, Glenda Dhenyfer Rocha ${ }^{4}$

TIBÉRIO, Lara de Vargas ${ }^{5}$

ROSI, Maria Eduarda Almagro ${ }^{6}$

FREIRE, Matheus Rodrigues Sardinha Drumond ${ }^{7}$

\footnotetext{
${ }^{1}$ Discente do curso de Medicina do Centro Universitário UNINTA, Sobral - CE.

${ }^{2}$ Médico Psiquiatra e docente do curso de Medicina do Centro Universitário INTA UNINTA.

${ }^{3}$ Discente do curso de Medicina do Centro Universitário UniFacid.

${ }^{4}$ Discente do curso de Medicina do Centro Universitário UNINTA, Sobral - CE.

${ }^{5}$ Discente do curso de Medicina do Centro Universitário do Espírito Santo - Campus Colatina.

${ }^{6}$ Discente do curso de Medicina do Centro Universitário do Espírito Santo - Campus Colatina.

${ }^{7}$ Discente do curso de Medicina do Centro Universitário do Espírito Santo - Campus Colatina.
} 
OLIVEIRA, Nikhole ${ }^{8}$

MELO, Rayana Cibelle Viana de ${ }^{9}$

VELOSO, Thamires Mendes 10

RIBEIRO, Yana Sarah Fernandes Souza. Et al. Implicações da Síndrome de Burnout em profissionais da saúde durante a pandemia de COVID-19: Uma revisão integrativa. Revista Científica Multidisciplinar Núcleo do Conhecimento. Ano 05, Ed. 10, Vol. 06, pp. 87-107. Outubro de 2020. ISSN: 2448-0959, Link de acesso:

\section{RESUMO}

Objetivo: Identificar, na literatura atual, as principais implicações e consequências da Síndrome de Burnout em Profissionais de Saúde. Métodos: Trata-se de uma revisão bibliográfica do tipo integrativa, com abordagem qualitativa e objetivo descritiva de estudos nacionais e internacionais. Para o desenvolvimento deste trabalho, foi realizada pesquisa bibliográfica na base de dados PubMed, na qual foram priorizados estudos publicados a partir de 2019 e artigos integralmente disponíveis que retratassem a questão de pesquisa, anexados em quaisquer idiomas. 20 artigos foram selecionados para leitura na íntegra e constituem esta revisão de literatura. Resultados: $47,36 \%$ dos estudos apontaram que a tensão e a demanda entre os profissionais de saúde aumentaram durante a pandemia da COVID-19, podendo predispor ao surgimento de quadros de Burnout. Dentro dos sintomas de esgotamento, os estudos frisaram, primordialmente, a exaustão cognitiva, ansiedade, estresse, sobrecarga psicológica, culpa, medo e insônia. As implicações mais relatadas nos estudos foram: sintomas depressivos, transtornos de ansiedade e

\footnotetext{
${ }^{8}$ Discente do curso de Medicina do Centro Universitário do Espírito Santo - Campus Colatina.

${ }^{9}$ Discente do curso de Medicina do Centro Universitário UNINTA, Sobral - CE.

${ }^{10}$ Discente do curso de Medicina do Centro Universitário do Espírito Santo - Campus Colatina.
} 
reações de estresse pós-traumático. Conclusão: Diante das complicações relacionadas ao esgotamento profissional, urge a adoção de medidas preventivas de apoio psicológico à equipe de saúde no período da pandemia de COVID-19 e continuamente, com o fito de melhorar a assistência e reduzir os prejuízos do Burnout.

Palavras-chave: Burnout, esgotamento profissional, profissionais de saúde, COVID19.

\section{INTRODUÇÃO}

Síndrome de Burnout é um acometimento psicológico que surge em resposta a fatores estressores interpessoais ocorridos durante a jornada de trabalho, causando exaustão física e mental associados ao estresse recorrente. Esta condição envolve uma sensação crescente e contínua de despersonalização, além de provocar um intenso esgotamento emocional e reduzir a sensação de realização pessoal vinculada ao trabalho (MASLACH e LEITER, 2016). Observou-se que os indivíduos com Burnout tendem a perder, progressivamente, a capacidade de realizar de maneira efetiva suas atividades laborais, tanto pelo desenvolvimento de ceticismo acerca do valor do seu trabalho, quanto pelo surgimento de dúvidas quanto à capacidade de desempenhá-lo (MASLACH, 1996).

Sabe-se que o esgotamento profissional tende a ser mais evidente em áreas de serviços humanos, como entre profissionais de saúde, assistentes sociais, psicoterapeutas, professores e juristas. Isto pode ser explicado pela alta demanda técnica e emocional que está atrelada a estas profissões, que comumente exigem esforços demasiados em cuidados, trocas sociais, empatia e compaixão (SCHAUFELI et al., 2006). Desta forma, por estar mais relacionada a profissões atreladas a valores e compromissos sociais, a exposição ao "esgotamento do cuidador", a insatisfação e a perda de compromisso nas relações pessoais e profissionais podem desencadear Transtorno de Estresse Pós-Traumático, ansiedade, depressão, ideações suicidas e dependência ou abuso de substâncias químicas (EL-HAGE et al., 2020). 
Nesta perspectiva, muitas intervenções estão sendo realizadas para promover o bem estar do indivíduo e reduzir as consequências advindas do Burnout em profissionais de saúde, como redução da carga de trabalho, emprego com horários de trabalho flexíveis e adoção de registro eletrônico de saúde, atenção plena e treinamento pessoal. Estas estratégias, associadas à psicoterapia, ajudam os indivíduos a serem mais colaborativos no ambiente de trabalho, possibilitando um aumento na produtividade, bem como uma maior qualidade de vida (DYRBE, 2019; PANAGIOTI, 2017; SHANAFELT, 2017).

No que tange à Síndrome de Burnout em profissionais de saúde, é sabido que, diante da crise de recursos associada à pandemia de COVID-19, muitos destes indivíduos têm sofrido, diariamente, estresses físicos, mentais e sociais. Fatores como a escassez de equipamentos de proteção individual e a difusão de informações mal interpretadas, dúbias ou mentirosas acerca da doença, por exemplo, têm contribuído para o avanço do esgotamento profissional. Percebe-se que o trabalhador que está em posição de cuidador, neste cenário, está mais suscetível a cometer imprudência e/ou negligência médica, a recorrer em ausências frequentes e, até mesmo, a cogitar aposentadoria precoce, consequências estas que são totalmente contrárias aos interesses do combate à pandemia de infecção por Sars-CoV-2 (RESTAURI et al., 2020). Neste sentido, emerge o seguinte questionamento: diante do cenário pandêmico atual, quais seriam as implicações da Síndrome de Burnout em profissionais de saúde?

Portanto, partindo da premissa de que as demandas emocionais e estruturais dos profissionais de saúde cresceram em níveis alarmantes durante a pandemia de COVID-19, esta pesquisa tem por objetivo primordial descrever, analisar e discutir as complicações decorrentes da Síndrome de Burnout nos trabalhadores que estão inseridos neste contexto. 


\section{DESENVOLVIMENTO}

\section{METODOLOGIA}

Trata-se de uma revisão bibliográfica do tipo integrativa, com abordagem qualitativa, de procedimento bibliográfico, natureza aplicada e objetivo descritivo de estudos nacionais e internacionais. Este método de pesquisa é abrangente, visto que é fundamentado em revisar referências bibliográficas com a finalidade de integrar as principais considerações observadas pelos autores.

A pesquisa foi realizada utilizando a base de dados PubMed e seus sites aliados. Primeiramente, foi feita uma análise dos descritores relevantes para o tema, por meio do vocabulário disponível no MeSH (Medical Subject Headings) sendo esses: "Burnout", "Síndrome de Burnout", "Profissionais da Saúde" e "Infecções por Coronavírus". Estes descritores, também traduzidos em inglês e espanhol, configuraram a seguinte fórmula de busca: "(Burnout OR Agotamiento Profesional OR Esgotamento Profissional OR Occupational Burnout OR Professional Burnout OR Burn out OR Burn out Syndrome OR Burn-out OR Burn-out Syndrome OR Burnout Syndrome OR Caregiver Burnout OR Caregiver Exhaustion OR Psychological Burn out OR Psychological Burn-out OR Psychological Burnout) AND (Prestadores de Cuidados de Saúde OR Profissionais da Saúde OR Profissionais de Saúde OR Profissional da Saúde OR Profissional de Saúde OR Trabalhador da Saúde OR Trabalhador de Saúde OR Trabalhadores da Saúde OR Trabalhadores de Saúde OR Personal de Salud OR Pessoal de Saúde OR Health Personnel OR Médicos OR Enfermeiros) AND (Coronavirus Infections OR Infecciones por Coronavírus OR Infecções por Coronavírus OR 2019 novel coronavírus Pneumonia OR 2019-novel coronavírus Pneumonia OR 2019 novel coronavírus Epidemic OR 2019 novel coronavírus Outbreak OR 2019 novel coronavírus Pandemic OR 2019-nCoV Acute Respiratory Disease OR 2019-nCoV Epidemic OR 2019-nCoV Outbreak OR 2019nCoV Pandemic OR 2019-nCoV Pneumonia OR 2019-novel coronavírus (2019-nCoV) Infection 2019new coronavírus Epidemic OR 201920 China Pneumonia Outbreak OR 201920 Wuhan coronavírus Outbreak OR COVID-19 OR Coronavirus Infection OR 
MERS (Middle East Respiratory Syndrome) OR Middle East Respiratory Syndrome OR Novel Coronavirus Pneumonia OR Wuhan Seafood Market Pneumonia OR Wuhan coronavirus Epidemic OR Wuhan coronavirus Infection OR Wuhan coronavirus Outbreak OR Wuhan coronavirus Pandemic OR Wuhan coronavirus Pneumonia)".

Por conseguinte, de acordo com os resultados obtidos a partir da fórmula de busca já mencionada, foram encontrados 58 artigos no total. Como critérios de inclusão foram utilizados: artigos indexados na base de dados PubMed a partir do ano 2019; artigos integralmente disponíveis que retratassem a questão de pesquisa deste trabalho e artigos em todos os idiomas. Os critérios de exclusão, por sua vez, foram: artigos que não faziam jus ao propósito da pesquisa; artigos publicados antes de 2019 e artigos de revisão sistemática.

Finalmente, restaram 19 artigos, que foram analisados pela leitura do título e do resumo com o intuito de correlacioná-los com a demanda da pesquisa. Diante disso, foi elaborada uma tabela contendo as principais informações dos artigos que foram revisados na pesquisa, sendo essas: título, autores, ano de publicação, país de origem e principais resultados encontrados.

Vale salientar que a pesquisa não apresenta caráter prático, o que dispensa, portanto, a necessidade de submissão ao Comitê de Ética em Pesquisa (CEP).

Tabela 1: Principais Resultados dos Artigos Selecionados para Pesquisa

\begin{tabular}{|c|c|c|c|}
\hline & Título & $\begin{array}{l}\text { Autores, ano de } \\
\text { publicação e país }\end{array}$ & Principais Resultados \\
\hline ARTIGO & Coronavirus & Fessell & exercício \\
\hline I & $\begin{array}{l}2019 \text { (COVID-19) and } \\
\text { Beyond: Micropractices } \\
\text { for Burnout Prevention } \\
\text { and Emotional Wellness }\end{array}$ & $\begin{array}{l}\text { Cherniss, } 2020 \text {, } \\
\text { Estados Unidos }\end{array}$ & $\begin{array}{l}\text { micropráticas, tais como a } \\
\text { respiração diafragmática e } \\
\text { nomeação das emoções } \\
\text { têm demonstrado ajudar } \\
\text { na prevenção da } \\
\text { síndrome de burnout, }\end{array}$ \\
\hline
\end{tabular}




\begin{tabular}{|c|c|c|c|}
\hline & & & $\begin{array}{l}\text { estresse e ansiedade em } \\
\text { profissionais da saúde. }\end{array}$ \\
\hline $\begin{array}{l}\text { ARTIGO } \\
\text { II }\end{array}$ & $\begin{array}{l}\text { [Health professionals } \\
\text { facing the coronavirus } \\
\text { disease } 2019 \text { (COVID- } \\
\text { 19) pandemic: What are } \\
\text { the mental health risks?] }\end{array}$ & $\begin{array}{l}\text { El-Hage et al., } \\
\text { 2020, França }\end{array}$ & $\begin{array}{l}\text { Observou-se o aumento } \\
\text { da prevalência de } \\
\text { doenças como síndrome } \\
\text { de bornout, ansiedade e } \\
\text { estresse nos cuidadores } \\
\text { da linha de frente na } \\
\text { pandemia de SARS-CoV- } \\
\text { 2. }\end{array}$ \\
\hline $\begin{array}{l}\text { ARTIGO } \\
\text { III }\end{array}$ & $\begin{array}{l}\text { Clinician Wellness } \\
\text { During the COVID-19 } \\
\text { Pandemic: } \\
\text { Extraordinary Times } \\
\text { and Unusual } \\
\text { Challenges for the } \\
\text { Allergist/lmmunologist }\end{array}$ & $\begin{array}{ll}\text { Bansal et al., } \\
\text { 2020, } & \text { Estados } \\
\text { Unidos } & \end{array}$ & $\begin{array}{l}\text { Profissionais de saúde } \\
\text { atuantes na pandemia de } \\
\text { SARS-CoV-2 têm } \\
\text { abusado do uso de drogas } \\
\text { e álcool e, por } \\
\text { consequência, têm } \\
\text { apresentado um risco } \\
\text { aumentado de insônia, } \\
\text { depressão e suicídio. }\end{array}$ \\
\hline $\begin{array}{l}\text { ART } \\
\text { IV }\end{array}$ & $\begin{array}{l}\text { Mitigating the } \\
\text { Psychological Impact of } \\
\text { COVID-19 on } \\
\text { Healthcare } \\
\text { Digital Workers: A } \\
\text { Package }\end{array}$ & $\begin{array}{l}\text { Blake et al., 2020, } \\
\text { Reino Unido }\end{array}$ & $\begin{array}{l}\text { Criação de uma } \\
\text { ferramenta de apoio } \\
\text { online que visa auxiliar o } \\
\text { bem-estar psicológico dos } \\
\text { profissionais da saúde } \\
\text { durante a pandemia e } \\
\text { após o seu término. }\end{array}$ \\
\hline $\begin{array}{l}\text { ARTIGO } \\
\mathrm{V}\end{array}$ & $\begin{array}{l}\text { A Comparison of } \\
\text { Burnout } \quad \text { Frequency } \\
\text { Among Oncology }\end{array}$ & $\begin{array}{l}\text { Wu et al., } 2020 \text {, } \\
\text { Estados Unidos }\end{array}$ & $\begin{array}{l}\text { O estudo mostrou uma } \\
\text { menor incidência da } \\
\text { síndrome de burnout na } \\
\text { equipe médica que } \\
\text { trabalha na linha de frente }\end{array}$ \\
\hline
\end{tabular}




\begin{tabular}{|c|c|c|c|}
\hline & $\begin{array}{l}\text { Physicians and Nurses } \\
\text { Working on the } \\
\text { Frontline and Usual } \\
\text { Wards During the } \\
\text { COVID-19 Epidemic in } \\
\text { Wuhan, China }\end{array}$ & & $\begin{array}{l}\text { da epidemia de COVID-19 } \\
\text { em comparação com as } \\
\text { equipes que trabalham } \\
\text { nas enfermarias habituais. }\end{array}$ \\
\hline $\begin{array}{l}\text { ARTIGO } \\
\text { VI }\end{array}$ & $\begin{array}{l}\text { Battle Buddies: Rapid } \\
\text { Deployment of a } \\
\text { Psychological } \\
\text { Resilience Intervention } \\
\text { for Health Care Workers } \\
\text { During the COVID-19 } \\
\text { Pandemic }\end{array}$ & $\begin{array}{l}\text { Albott et al., 2020, } \\
\text { Estados Unidos }\end{array}$ & $\begin{array}{l}\text { Implementação do } \\
\text { sistema Battle Buddy do } \\
\text { exército americano dos } \\
\text { Estados Unidos frente ao } \\
\text { combate à pandemia de } \\
\text { COVID-19 pode auxiliar } \\
\text { reduzindo as pressões e } \\
\text { sofrimentos vivenciados } \\
\text { pelos profissionais da } \\
\text { saúde, relacionados ao } \\
\text { ambiente de trabalho. }\end{array}$ \\
\hline $\begin{array}{l}\text { ARTIGO } \\
\text { VII }\end{array}$ & $\begin{array}{l}\text { Burnout and } \\
\text { Posttraumatic Stress } \\
\text { Disorder in the } \\
\text { Coronavirus Disease } \\
2019 \text { (COVID-19) } \\
\text { Pandemic: Intersection, } \\
\text { Impact, and } \\
\text { Interventions }\end{array}$ & $\begin{array}{l}\text { Restauri } \\
\text { Sheridan, 2020, } \\
\text { Estados Unidos }\end{array}$ & $\begin{array}{l}\text { Exposição a experiências } \\
\text { traumáticas } \\
\text { estressantes tem a } \\
\text { capacidade de promover o } \\
\text { aparecimento em } \\
\text { sequência de transtorno } \\
\text { de estresse agudo, } \\
\text { transtorno de estresse } \\
\text { pós-traumático } \\
\text { síndrome de burnout. }\end{array}$ \\
\hline $\begin{array}{l}\text { ARTIGO } \\
\text { VIII }\end{array}$ & $\begin{array}{l}\text { Mental health } \\
\text { circumstances among } \\
\text { health care workers and } \\
\text { general public under the }\end{array}$ & $\begin{array}{l}\text { Nochaiwong et } \\
\text { al., 2020, Estados } \\
\text { Unidos }\end{array}$ & $\begin{array}{l}\text { Profissionais da saúde } \\
\text { que tinham um contato } \\
\text { direto com os pacientes } \\
\text { infectados com SARS- }\end{array}$ \\
\hline
\end{tabular}




\begin{tabular}{|c|c|c|c|}
\hline & $\begin{array}{l}\text { pandemic situation of } \\
\text { COVID-19 } \\
\text { COVID-19) }\end{array}$ & & $\begin{array}{l}\text { CoV-2 apresentaram um } \\
\text { maior risco para } \\
\text { desenvolver depressão, } \\
\text { ansiedade e angústia. }\end{array}$ \\
\hline $\begin{array}{l}\text { ARTIGO } \\
\text { IX }\end{array}$ & $\begin{array}{l}\text { SAGES primer for } \\
\text { taking care of yourself } \\
\text { during and after the } \\
\text { COVID- } 19 \text { crisis }\end{array}$ & $\begin{array}{l}\text { Dort et al., 2020, } \\
\text { Estados Unidos }\end{array}$ & $\begin{array}{l}\text { Aumento do medo e } \\
\text { estresse em profissionais } \\
\text { da saúde atuantes na } \\
\text { pandemia de COVID-19, } \\
\text { causados pelo risco de } \\
\text { infecção própria e } \\
\text { transmissão para seus } \\
\text { familiares. }\end{array}$ \\
\hline $\begin{array}{l}\text { ARTIGO } \\
x\end{array}$ & $\begin{array}{l}\text { Mental health among } \\
\text { otolaryngology resident } \\
\text { and attending } \\
\text { physicians during the } \\
\text { COVID-19 pandemic: } \\
\text { National study }\end{array}$ & $\begin{array}{l}\text { Civantos et al., } \\
2020, \quad \text { Estados } \\
\text { Unidos }\end{array}$ & $\begin{array}{l}\text { Preocupação com a } \\
\text { saúde mental de } \\
\text { profissionais da saúde, } \\
\text { em especial } \\
\text { otorrinolaringologistas, } \\
\text { pelo aumento da } \\
\text { ansiedade e estresse } \\
\text { gerados pelo caráter } \\
\text { essencial de seus tipos de } \\
\text { procedimentos. }\end{array}$ \\
\hline $\begin{array}{l}\text { ARTIGO } \\
X I\end{array}$ & $\begin{array}{l}\text { Addressing General } \\
\text { Surgery Residents' } \\
\text { Concerns in the Early } \\
\text { Phase of the COVID-19 } \\
\text { Pandemic }\end{array}$ & $\begin{array}{l}\text { He et al., 2020, } \\
\text { Estados Unidos }\end{array}$ & $\begin{array}{l}\text { Organização dos serviços } \\
\text { como delegação de } \\
\text { tarefas e limite de horas } \\
\text { no trabalho, além do } \\
\text { diálogo com a família dos } \\
\text { profissionais da saúde, } \\
\text { apresenta a capacidade } \\
\text { de reduzir a ansiedade, } \\
\text { fadiga, estresse e }\end{array}$ \\
\hline
\end{tabular}




\begin{tabular}{|c|c|c|c|}
\hline & & & $\begin{array}{l}\text { esgotamento dos } \\
\text { profissionais da saúde } \\
\text { durante a crise de COVID- } \\
19 .\end{array}$ \\
\hline $\begin{array}{l}\text { ARTIGO } \\
\text { XII }\end{array}$ & $\begin{array}{l}\text { Covid-19: doctors must } \\
\text { take control of their } \\
\text { wellbeing }\end{array}$ & $\begin{array}{l}\text { Simons e } \\
\text { Baldwin, 2020, } \\
\text { Reino Unido }\end{array}$ & $\begin{array}{l}\text { O estudo evidenciou que } \\
\text { profissionais saúde têm } \\
\text { um sentimento de que a } \\
\text { sua vida e a de seus } \\
\text { familiares não é } \\
\text { valorizada, devido às } \\
\text { condições de trabalho } \\
\text { encontradas durante o } \\
\text { enfrentamento da COVID- } \\
\text { 19. }\end{array}$ \\
\hline $\begin{array}{l}\text { ARTIGO } \\
\text { XIII }\end{array}$ & $\begin{array}{l}\text { COVID19 pandemic } \\
\text { impacts on anxiety of } \\
\text { French urologist in } \\
\text { training: Outcomes from } \\
\text { a national survey }\end{array}$ & $\begin{array}{l}\text { Abdessater et al., } \\
\text { 2020, França }\end{array}$ & $\begin{array}{l}\text { Resultado psicológico } \\
\text { desagradável em } \\
\text { profissionais da saúde } \\
\text { que enfrentam a } \\
\text { pandemia de coronavírus, } \\
\text { em virtude da sensação } \\
\text { de não estar conseguindo } \\
\text { prover um serviço eficaz, } \\
\text { além do sentimento de } \\
\text { falta de segurança (pela } \\
\text { escassez de } \\
\text { equipamentos de } \\
\text { proteção individual). }\end{array}$ \\
\hline $\begin{array}{l}\text { ARTIGO } \\
\text { XIV }\end{array}$ & $\begin{array}{l}\text { Psychosocial burden of } \\
\text { healthcare } \\
\text { professionals in times of } \\
\text { COVID-19 - a survey }\end{array}$ & $\begin{array}{l}\text { Zerbini } \\
\text { al.,2020, } \\
\text { Alemanha }\end{array}$ & $\begin{array}{l}\text { Os profissionais da saúde } \\
\text { que possuem um contato } \\
\text { maior com pacientes } \\
\text { contaminados pela }\end{array}$ \\
\hline
\end{tabular}




\begin{tabular}{|c|c|c|c|}
\hline & $\begin{array}{l}\text { conducted at the } \\
\text { University Hospital } \\
\text { Augsburg }\end{array}$ & & $\begin{array}{lr}\text { COVID-19 } & \text { apresentaram } \\
\text { um maior } & \text { desgaste } \\
\text { psicossocial } & \text { quando } \\
\text { comparados } & \text { aos } \\
\text { profissionais } & \text { que } \\
\text { trabalham em enfermarias } \\
\text { regulares. }\end{array}$ \\
\hline $\begin{array}{l}\text { ARTIGO } \\
X V\end{array}$ & $\begin{array}{l}\text { Impact of COVID-19 on } \\
\text { the mental health of } \\
\text { surgeons and coping } \\
\text { strategies }\end{array}$ & $\begin{array}{l}\text { Balasubramanian } \\
\text { et al., 2020, Reino } \\
\text { Unido }\end{array}$ & $\begin{array}{l}\text { A promoção de medidas } \\
\text { como sistema de troca de } \\
\text { turnos e pausas do } \\
\text { ambiente de trabalho, } \\
\text { além do } \\
\text { acompanhamento com } \\
\text { psicólogo, pode preservar } \\
\text { a saúde mental dos } \\
\text { profissionais da saúde } \\
\text { durante a pandemia de } \\
\text { CoviD-19. }\end{array}$ \\
\hline $\begin{array}{l}\text { ARTIGO } \\
X V I\end{array}$ & $\begin{array}{l}\text { The Psychosocial } \\
\text { Impact of COVID-19 on } \\
\text { health care workers }\end{array}$ & $\begin{array}{l}\text { Rodríguez } \\
\text { Sánchez ,2020, } \\
\text { Brasil }\end{array}$ & $\begin{array}{l}\text { Em razão do isolamento } \\
\text { social, além do medo de } \\
\text { transmissão para seus } \\
\text { familiares, profissionais de } \\
\text { saúde possuem maiores } \\
\text { chances de desenvolver o } \\
\text { transtorno de estresse } \\
\text { pós-traumático e, por } \\
\text { consequência, a } \\
\text { Síndrome de Burnout. }\end{array}$ \\
\hline $\begin{array}{l}\text { ARTIGO } \\
x \text { VII }\end{array}$ & $\begin{array}{l}\text { Mental health crisis of } \\
\text { Japanese health care }\end{array}$ & $\begin{array}{ll}\text { Makino et al., } \\
\text { 2020, Estados } \\
\text { Unidos }\end{array}$ & $\begin{array}{l}\text { O estudo observou que o } \\
\text { aumento do número de } \\
\text { pessoas infectadas, o }\end{array}$ \\
\hline
\end{tabular}




\begin{tabular}{|c|c|c|c|}
\hline & $\begin{array}{l}\text { workers under COVID- } \\
19\end{array}$ & & $\begin{array}{l}\text { medo de falecer por conta } \\
\text { da contaminação pelo } \\
\text { vírus associados e o } \\
\text { aumento das tarefas } \\
\text { físicas no trabalho têm } \\
\text { desencadeado } \\
\text { comprometimentos na } \\
\text { saúde mental de } \\
\text { profissionais da saúde } \\
\text { que trabalham no } \\
\text { combate à COVID-19. }\end{array}$ \\
\hline $\begin{array}{l}\text { ARTIGO } \\
\text { XVIII }\end{array}$ & $\begin{array}{l}\text { Research on reducing } \\
\text { burnout in health care } \\
\text { workers in critical } \\
\text { situations such as the } \\
\text { COVID-19 outbreak }\end{array}$ & $\begin{array}{l}\text { Babamiri et al., } \\
\text { 2020, Irã }\end{array}$ & $\begin{array}{l}\text { O aumento do estresse, } \\
\text { esgotamento mental, } \\
\text { alterações no sono e } \\
\text { fadiga muscular em } \\
\text { profissionais da saúde } \\
\text { que trabalham contra a } \\
\text { pandemia de SARS-CoV- } \\
2 \text { ocorre em virtude do } \\
\text { aumento da demanda de } \\
\text { trabalho. }\end{array}$ \\
\hline $\begin{array}{l}\text { ARTIGO } \\
\text { XIX }\end{array}$ & $\begin{array}{l}\text { The Exacerbation of } \\
\text { Burnout During COVID- } \\
\text { 19: A Major Concern for } \\
\text { Nurse Safety }\end{array}$ & $\begin{array}{l}\text { Ross, } \quad 2020, \\
\text { Estados Unidos }\end{array}$ & $\begin{array}{l}\text { A grande } \\
\text { responsabilidade de seus } \\
\text { procedimentos } \\
\text { ambiente de trabalho, o } \\
\text { aumento de tarefas físicas } \\
\text { no atendimento e o } \\
\text { isolamento social foram } \\
\text { identificados como causas } \\
\text { do estresse mental dos } \\
\text { profissionais de saúde }\end{array}$ \\
\hline
\end{tabular}


Fonte: Próprio Autor.

\section{RESULTADOS}

Dentre os estudos analisados, 47,36\% $(n=9)$ apontaram que a tensão e a demanda entre os médicos aumentaram durante a pandemia da COVID-19, podendo predispor ao surgimento de quadros de Burnout. Dentro dos sintomas de esgotamento, os estudos frisaram, primordialmente, a exaustão cognitiva, ansiedade, estresse, sobrecarga psicológica, culpa, medo e insônia (EL-HAGE et al., 2020). Um estudo realizado na França indica que a rápida disseminação, a gravidade, as incertezas de conhecimento, os sentimentos de ameaça permanente e a morte entre os colegas profissionais de saúde aumentam o impacto no contexto psicológico destes cuidadores. Dentre os agravantes, foi destacada a falta de competência, insegurança em fazer escolhas e implementá-las, além da possibilidade veemente de infecção e transmissão para familiares (EL-HAGE et al., 2020). Os estudos revelaram, ainda, o sentimento de impotência em relação ao tratamento e à segurança por escassez de equipamentos de proteção individual, o que mostrou tendência a aumentar os níveis de estresse (SIMONS; BALDWIN, 2020). Estas perturbações possuem efeitos a longo prazo e duradouros, apresentando risco de cronicidade, o que prejudica a funcionalidade e qualidade de vida desses profissionais de saúde (ALBOTT et al., 2020).

Em 10,52\% (n=2) dos estudos, foi demonstrado que a realização de micro práticas, como entender momentos de higienização das mãos como uma oportunidade de autoconsciência e autogestão; escrever várias vezes durante a semana pelo menos 3 motivos pelos quais se é grato; nomear emoções afim de reconhecê-las e dominá-las; praticar a respiração diafragmática e compartilhar experiências profissionais no ambiente de trabalho pode ter benefícios sobre a melhora do bem-estar e contribuir para a prevenção da Síndrome de Burnout (FESSEL; CHERNISS, 2020). Estas micro 
práticas tem como objetivo gerenciar os aspectos emocionais e diminuir os níveis de estresse e medo (RESTAURI; SHERIDAN, 2020).

Como fator protetivo para o esgotamento, foi descrita a comunicação entre os cuidadores, o apoio social e a assistência logística, como o fornecimento de equipamentos de proteção individual para os cuidadores, a qual deve ser garantida pelas instituições prestadoras de serviços de saúde. Além disso, também se destaca a realização de pausas no trabalho, o seguimento das regras higienodietéticas diárias como dormir, realizar prática de atividade física, reduzir da exposição a telas e meios de comunicação, bem como afastar-se do consumo de álcool e drogas (EL-HAGE et al., 2020). Evidenciou-se benefício, até mesmo, em realizar o cancelamento de procedimentos eletivos, o que proporciona maior tempo fora do trabalho (CIVANTOS et al., 2020). Ainda dentro deste quesito, 15,78\% ( $n=3)$ dos estudos evidenciam recomendações para uso de pacotes de suporte digital, como aplicativos móveis, para promover o bem-estar psicológico em profissionais da saúde.

Em relação à comparação da saúde mental de médicos atuantes na primeira linha de exposição, durante a pandemia de SARS-CoV-2, e médicos de enfermarias habituais, houve algumas controvérsias. Para Wu et al. (2020), médicos da linha de frente sofrem menos sintomas de esgotamento, por possuírem maior sensação de controle de sua situação, controle este que está associado a um impulso de engajamento importante para prevenção destes sintomas. Porém, para El-hage et al., Nochaiwong et al. e Zerbini et al. (2020), médicos que tem contato direto com os pacientes portadores de COVID-19 possuem maior risco de sobrecarga psicológica, ansiedade, estresse, angústia, exaustão e insônia, com consequências a longo prazo.

Por fim, podemos afirmar que são necessárias estratégias de rastreamento de Síndrome de Burnout em cuidadores da primeira linha de atendimento e, também, a todos os profissionais de saúde. Exemplos de estratégias seriam o uso de questionários e prestação de assistência psicológica (EL-HAGE et al., 2020). 


\section{DISCUSSÃO}

A pandemia de Sars-CoV-2, inevitavelmente, colocou em voga os impactos e implicações da Síndrome de Burnout em profissionais da saúde, os quais, no cenário atual, têm sido mais comumente acometidos por esta condição. Além da Síndrome de Esgotamento Profissional, estes indivíduos estão sujeitos a outros distúrbios psicológicos derivados de altas taxas de estresse cotidiano, tais como ansiedade e transtorno de estresse pós-traumático (RESTAURI E SHERIDAN, 2020). De acordo com Luceño-Moreno et al. (2020), durante a epidemia de Síndrome Respiratória Aguda Grave (SARS) de 2002, sintomas como raiva, perda de motivação no trabalho, dificuldade de concentração e insônia também foram comumente relatados e identificados em profissionais do setor de emergência. Entretanto, nem todos os indivíduos que foram expostos a estes impactos negativos e situações críticas desenvolveram os sintomas. Segundo o autor, resiliência e altos níveis de confiança foram fatores protetores para lidar com toda a carga de estresse direcionada aos profissionais, o que resultou na sensação de controle diante da adversidade e, com isso, menores prejuízos no ambiente de trabalho e no contexto pessoal.

Segundo Zerbini et al. (2020), os principais motivos que causam estresse e preocupações nos profissionais de saúde que lidam cotidianamente com a pandemia atual da COVID-19 são o aumento da carga horária no trabalho, mudanças repentinas e frequentes na equipe e aumento dos conflitos entre colegas, além da apreensão com o futuro, pelos riscos de colapso no sistema de saúde e na economia mundial. Da mesma forma, estudo sul-africano de 2015 demonstrou que exaustão emocional, insatisfação com pagamento ou com a supervisão do setor, bem como com as condições operacionais ou com os colegas de trabalho e ineficiência da comunicação foram associados a maiores taxas de desenvolvimento de Burnout entre enfermeiros (KHAMIZA et al., 2015).

No contexto atual de disseminação do SARS-CoV-2, mesmo profissionais da saúde que não são especializados em doenças infecciosas foram designados para auxiliar no combate ao vírus. Neste contexto, pesquisas que compararam a frequência da síndrome de Burnout em profissionais infectologistas em comparação a profissionais 
que atuaram em outras especialidades durante a pandemia demonstraram que participantes da linha de frente possuíam uma frequência significativamente menor de Burnout e estão menos preocupados em se infectar, apesar de trabalharem diretamente com pacientes contaminados. Algumas hipóteses são sugeridas a respeito desta conclusão, sendo elas: em comparação com trabalhadores de outras áreas da saúde, em profissionais especializados em infectologia fora observada maior sensação de controle no ambiente de trabalho e maior proximidade da tomada de decisões-chave o que, portanto, revelou obtenção de maior acurácia nas informações absorvidas. Por sua vez, em profissionais de saúde sem contato direto com o enfrentamento da COVID-19, notou-se menor controle sobre novas políticas, protocolos e procedimentos de segurança, associados à sensação de que a pandemia de SARS-CoV-2 poderia impactar o seu ambiente de trabalho a qualquer momento, independentemente das medidas de segurança adotadas (WU et al., 2020).

No que tange ao sentimento de culpa e vergonha, estes têm sido muito presentes nas equipes de saúde durante o período da atual pandemia de SARS-CoV-2, sobretudo, devido aos limitados recursos de equipamentos de proteção individual disponibilizados. Uma grande parcela dos profissionais de saúde relata a preocupação exagerada quanto à segurança sanitária e quanto à chance de transmissão do vírus para familiares, o que comumente tem desencadeado culpa e remorso (SIMONS E BALDWIN, 2020). Outrossim, fora observado que profissionais da saúde submetidos a ambientes hostis e cargas exorbitantes de estresse, como o do cenário atual, foram mais propensos a desenvolver quadros de ansiedade, depressão, insônia, sintomas somáticos e disfunção social, atrelados ao esgotamento emocional (KHAMISA et al., 2015).

Pesquisa promovida por Sun et al. avaliou as consequências da sobrecarga psicológica de profissionais atuantes no enfrentamento da pandemia de COVID-19. Neste estudo, foi identificada, nestes trabalhadores, a predominância de emoções negativas como fadiga e desconforto, que foram promovidas pela alta intensidade e extenuante carga horária de trabalho. Além disso, foi evidenciada a maximização do medo, da ansiedade e da preocupação com os pacientes e familiares. Esta conclusão 
é corroborada por estudo publicado em agosto de 2020, o qual demonstrou que a exaustão emocional presente em enfermeiros, durante a pandemia de infecção pelo SARS-CoV-2, decorre principalmente da constante conscientização e vigilância quanto aos procedimentos de controle sanitários, assim como do frequente desgaste físico dos equipamentos de proteção e do isolamento social preconizado pelos governos (ROSS, 2020).

Vale salientar que micro práticas realizadas no ambiente profissional têm demostrado benefícios na prevenção e terapia da Síndrome de Burnout, destacando o exercício da respiração diafragmática (FESSEL; CHERNISS, 2020). Conforme estudos abordados por Hopper et al., o indivíduo, ao respirar de modo profundo e expandindo os pulmões para dentro do diafragma, possivelmente pode desencadear uma diminuição do estresse fisiológico e psicológico, condições que podem acarretar a Síndrome do Esgotamento Profissional. Esta prática também evidenciou uma melhora nos níveis pressóricos e na sub escala de estresse da Escala de Depressão, Ansiedade e Estresse (DASS-21). Neste sentido, ferramentas digitais desenvolvidas no período da pandemia de COVID-19 também surgiram como importantes mecanismos de apoio e bem-estar psicológico aos profissionais da saúde. A título de exemplo, fora desenvolvida uma plataforma online criada inicialmente para provedores de cuidados em saúde do Reino Unido que visa, justamente, proporcionar suporte emocional durante e após a pandemia. Este trabalho apresentou significativa procura e aplicabilidade, fatores que justificam a necessidade da utilização destes instrumentos virtuais no cenário atual (BLAKE et al., 2020).

Outra proposta de manejo é a promoção de condições ocupacionais que promovam baixo risco de infecção por COVID-19, sendo necessária uma maior disponibilidade de testes diagnósticos e equipamentos de proteção individual aos profissionais de saúde. Dessa forma, seria garantido a estes trabalhadores uma maior segurança, tranquilidade e, também, menor preocupação quanto a transmissão do vírus para entes queridos. Neste sentido, tem sido demonstrada eficácia através da criação de cronogramas com tempo-limite de trabalho e rotatividade de profissionais, o que possibilita redução da fadiga, do estresse e do esgotamento laboral. (HE et al., 2020). 
Esta alternativa teve seu benefício comprovado pelo estudo qualitativo realizado por Sun et al., no qual foram avaliadas as consequências psicológicas de cuidadores de pacientes com COVID-19.

\section{CONSIDERAÇÕES FINAIS}

Os dados revisados sobre a Síndrome de Burnout nos profissionais de saúde inseridos no contexto da pandemia de infecção por SARS-CoV-2 demonstram que as situações de excessivo esforço mental e físico a que têm sido submetidos podem desencadear dificuldades de concentração, sentimentos de fracasso e insegurança, cefaleia frequente e dores musculares, bem como transtornos de depressão e ansiedade. Estes agravos evidenciam a importância da atenção e prevenção para posterior tratamento da síndrome.

Logo, faz-se necessário desenvolver ações preventivas e ativas de melhora das condições de trabalho, tais como: incentivar a prática de exercício físico antes da atividade laboral, evitar a alta demanda de trabalho, fazer pausas esporádicas e ter um apoio psicológico profissional. Estas atitudes que objetivam avanços na qualidade de vida dos profissionais refletem na melhoria da prestação de assistência ao paciente.

Portanto, embora já existam diversas terapias e medidas de controle de complicações atreladas à Síndrome de Burnout, tais como ansiedade, estresse e síndromes depressivas, ainda se faz necessária a conscientização acerca da importância destas ações no ambiente hospitalar e ambulatorial. Urge, também, o fornecimento de apoio psicológico à equipe de saúde no período da pandemia de COVID-19 e continuamente, com o fito de maximizar a aplicabilidade e o sucesso destas condutas.

\section{REFERÊNCIAS}

ALBOTT, Cristina et al. Battle Buddies: Rapid Deployment of a Psychological Resilience Intervention for Health Care Workers during the COVID-19 Pandemic. 
Anesthesia and Analgesia, [S. I.], v. XXX, n. Xxx, p. 43-54, 2020. DOI: 10.1213/ANE.0000000000004912.

BABAMIRI, Mohammad; ALIPOUR, Nasim; HEIDARIMOGHADAM, Rashid. Research on reducing burnout in health care workers in critical situations such as the COVID-19 outbreak. Work (Reading, Mass.), v. 66, n. 2, p. 379-380, 2020. DOI: 10.3233/WOR203189.

BALASUBRAMANIAN, Anusha et al. Impact of COVID-19 on the mental health of surgeons and coping strategies. Head and Neck, [S. I.], v. 42, n. 7, p. 1638-1644, 2020. DOI: 10.1002/hed.26291.

BANSAL, $P$ et al. Clinician Wellness During the COVID-19 Pandemic: Extraordinary Times and Unusual Challenges for the Allergist/Immunologist. J Allergy Clin Immunol Pract. 2020;8(6):1781-1790.e3. DOI: 10.1016/j.jaip.2020.04.001

BLAKE, Holly et al. Mitigating the psychological impact of covid-19 on healthcare workers: A digital learning package. International Journal of Environmental Research and Public Health, [S. I.], v. 17, n. 9, 2020. DOI: 10.3390/ijerph17092997.

BUSELLI et al. Professional Quality of Life and Mental Health Outcomes among Health Care Workers Exposed to Sars-Cov-2 (Covid-19). Int. J. Environ. Res. Public Health, Itália, v. $17, \quad$ n. $6180, \quad$ p. 1-12, ago./2020. Disponível em: https://doi.org/10.3390/ijerph17176180. Acesso em: 7 set. 2020.

CIVANTOS, Alyssa M. et al. Mental health among otolaryngology resident and attending physicians during the COVID-19 pandemic: National study. Head and Neck, [S. I.], v. 42, n. 7, p. 1597-1609, 2020. DOI: 10.1002/hed.26292.

DORT, Jonathan et al. SAGES primer for taking care of yourself during and after the COVID-19 crisis. Surgical Endoscopy, [S. I.], v. 34, n. 7, p. 2856-2862, 2020. DOI: 10.1007/s00464-020-07631-3. Disponível em: https://doi.org/10.1007/s00464-02007631-3. 
DYRBE, Liselotte N.; SATELE, Daniel V. Effect of a Professional Coaching Intervention on the Well-being and Distress of Physicians: A Pilot Randomized Clinical Trial. JAMA Internal Medicine, [S. I.], v. 179, n. 10, p. 1406-1414, 5 ago. 2019.

EL-HAGE, W. et al. Les professionnels de santé face à la pandémie de la maladie à coronavirus (COVID-19): quels risques pour les santé mentale? L'Encéphale, [S. I.], v. 46, p. 73-80, 22 abr. 2020.

FESSELL, David; CHERNISS, Cary. Coronavirus Disease 2019 (COVID-19) and Beyond: Micropractices for Burnout Prevention and Emotional Wellness. Journal of the American College of Radiology, [S. I.], v. 17, n. 6, p. 746-748, 2020. DOI: 10.1016/j.jacr.2020.03.013.

HE, Katherine et al. Addressing General Surgery Residents' Concerns in the Early Phase of the COVID-19 Pandemic. Journal of Surgical Education, [S. I.], v. 77, n. 4, p. 735-738, 2020. DOI: 10.1016/j.jsurg.2020.04.003.

HOPPER, Susan I et al. Effectiveness of diaphragmatic breathing for reducing physiological and psychological stress in adults: a quantitative systematic review. JBI Database of Systematic Reviews, [s. I.], v. 17, ed. 9, p. 1855-1876, 2019. DOI: 10.11124/JBISRIR-2017-003848.

JOHNSON et al., Mental healthcare staff well being and burnout: A narrative review of trends, causes, implications, and recommendations for future interventions. Int $\mathbf{J}$ Mental Health Nurs, Austrália, v. 27, n. 1, p. 20-32, dez./2017. Disponível em: https://doi.org/10.1111/inm.12416. Acesso em: 7 set. 2020.

KHAMISA, Natasha et al. Work Related Stress, Burnout, Job Satisfaction and General Health of Nurses. International Journal of Environmental Research and Public Health, South Africa, p. 652-666, 12 jan. 2015.

LUCEÑO-MORENO et al. Symptoms of Posttraumatic Stress, Anxiety, Depression, Levels of Resilience and Burnout in Spanish Health Personnel during the COVID-19 Pandemic. nt. J. Environ. Res. Public Health, Espanha, v. 17, n. 5514, p. 1-25, 
jul./2020. Disponível em: https://doi.org/10.3390/ijerph17155514. Acesso em: 7 set. 2020.

MAKINO, Miyuki et al. Mental Health Crisis of Japanese Health Care Workers Under COVID-19. Psychological Trauma: Theory, Research, Practice, and Policy, [S. I.], v. 12, p. 136-137, 2020. DOI: 10.1037/tra0000819.

MARQUES, M. M. et al. The effect of profession on burnout in hospital staff,. Occupational Medicine, Oxford, v. 68, n. 3, p. 207-210, mai./2018. Disponível em: https://doi.org/10.1093/occmed/kqy039. Acesso em: 7 set. 2020.

MASLACH, Christina et al. Maslach Burnout Inventory Manual. Consulting Psychologists Press, EUA, n. 3, p. 191-218, 1996.

MASLACH, Christina et al. Understanding the burnout experience: recent research and its implications for psychiatry. World Psychiatry, USA, v. 2, n. 15, p. 103-111, 20 jun. 2016.

MESSMAN, G et al. Psychosocial burden of healthcare professionals in times of COVID-19 - a survey conducted at the University Hospital Augsburg. German Medical Science, Germany , v. 18, n. 5, p. 1-9, jul./2020. Disponível em: http://creativecommons.org/licenses/by/4.0/. Acesso em: 7 set. 2020.

NOCHAIWONG, Surapon et al. Mental health circumstances among health care workers and general public under the pandemic situation of COVID-19 (HOMECOVID-19). Medicine, [S. I.], v. 99, n. 26, p. e20751, 2020. DOI: 10.1097/MD.0000000000020751.

PANAGIOTI, Maria et al. Controlled Interventions to Reduce Burnout in Physicians: A Systematic Review and Meta-analysis. JAMA Internal Medicine, United Kingdom, p. 1-11, 5 dez. 2016. 
RASMUSSEN, Sonja A, and Denise J Jamieson. Coronavirus Disease 2019 (COVID19) and Pregnancy: Responding to a Rapidly Evolving Situation. Obstetrics and gynecology, vol. 135,5 (2020): 999-1002. doi:10.1097/AOG.0000000000003873

RESTAURI, Nicole; SHERIDAN, Alison D. Burnout and Posttraumatic Stress Disorder in the Coronavirus Disease 2019 (COVID-19) Pandemic: Intersection, Impact, and Interventions. American College of Radiology, EUA, v. 17, n. 7, p. 921-926, jul. 2020.

RODRÍGUEZ, Bárbara Otonín. The Psychosocial Impact of COVID-19 on health care workers, International Brazilian Journal of Urology, v. 46, n. 1, p. 195-200, 2020. DOI: 10.1590/S1677-5538.IBJU. 2020.S124.

ROSS, Jacqueline. The Exacerbation of Burnout During COVID-19: A Major Concern for Nurse Safety. Journal of Perianesth Nurs, 2020 Aug;35(4):439-440. DOI: 10.1016/j.jopan.2020.04.001.

SCHAUFELI, Wilmar B.; LEITER, Michael P.; MASLACH, Christina. Burnout: 35 years of research and practice. Career Development International, The Netherlands, v. 14, n. 3, p. 204-220, 22 dez. 2008.

SHANAFELT, Tait D.; NOSEWORTHY, John H. Executive Leadership and Physician Well-being: Nine Organizational Strategies to Promote Engagement and Reduce Burnout. Mayo Clinic Proceedings, USA, v. 92, n. 1, p. 129-146, 18 nov. 2016.

SIMONS, Gemma; BALDWIN, David S. Covid-19: Doctors must take control of their wellbeing. The BMJ, [S. I.], v. 369, n. May, p. 2020, 2020. DOI: 10.1136/bmj.m1725. Disponível em: http://dx.doi.org/doi:10.1136/bmj.m1725.

SUN, Niuniu et al. A qualitative study on the psychological experience of caregivers of COVID-19 patients. American Journal of Infection Control, [s. I.], v. 48, ed. 6, p. 592-598, 2020. DOI: 10.1016/j.ajic.2020.03.018. 
UZZAMAN, Ashraf; $\mathrm{CHO}$, Seong H. Classification of hypersensitivity reactions. Allergy and Asthma Proceedings, EUA, v. 33, n. 14, p. 46-49, jul./2016. Disponível em: https://www.oceansidepubl.com/permission.htm. Acesso em: 20 ago. 2020.

WU, Y. et al. A Comparison of Burnout Frequency Among Oncology Physicians and Nurses Working on the Frontline and Usual Wards During the COVID-19 Epidemic in Wuhan, China. J Pain Symptom Manage. 2020;60(1): e60-e65.doi: 10.1016/j.jpainsymman.2020.04.008

WU, Y. et al. A Comparison of Burnout Frequency Among Oncology Physicians and Nurses Working on the Frontline and Usual Wards During the COVID-19 Epidemic in Wuhan, China. Journal of Pain and Symptom Management, China, v. 60, n. 1, p. 60-65, abr./2020. Disponível em: https://doi.org/10.1016/j.jpainsymman.2020.04.008. Acesso em: 7 set. 2020 .

ZERBINI, Giulia et al. Psychosocial burden of healthcare professionals in times of covid-19 - a survey conducted at the university hospital augsburg. GMS German Medical Science, [S. I.], v. 18, p. 1-9, 2020. DOI: 10.3205/000281.

Enviado: Setembro, 2020.

Aprovado: Outubro, 2020. 